\title{
Numerical Simulation of the Scalar Dissipation Rate in the Non-Premixed Turbulent Combustion
}

\author{
Y. F. Liu ${ }^{1,2}$, Y. T. Zhang ${ }^{1 *}$, H. S. Tian ${ }^{2}$, J. J. Qin ${ }^{3}$ \\ ${ }^{1}$ School of Mechanical and Vehicular Engineering, Beijing Institute of Technology, Beijing 100081, \\ China \\ ${ }^{2}$ School of Mechanical - electronic and Automobile Engineering, Beijing Institute of Civil Engineering \\ and Architecture, Beijing 100044, China \\ ${ }^{3}$ School of Mechanical, Electronic and Control Engineering, Beijing Jiaotong University, Beijing \\ 100044, China \\ Email: youtong@bit.edu.cn or liuyongfeng@bit.edu.cn
}

\begin{abstract}
To calculate the scalar dissipation rate in the non-premixed turbulent combustion different formulations for the conditional scalar dissipation rate are deducted. The scalar dissipation rate is an extremely important parameter in the turbulent combustion, since it alone accounts for effects of turbulent strain and stretch and represents the inverse of a characteristic diffusion time and as such it determines the rate of exchange of mass and energy. In the infinite unsteady one-dimensional laminar mixing layer, the similar coordinate and the complementary error function are introduced and the governed equation is transformed and the model 1 is got. However, the infinite unsteady one-dimensional laminar mixing layer is inadequate in describing the actual mixing processes taking place when a fucl jet is issued into the surrounding air. In the confined unsteady one-dimensional laminar mixing layer, a different set of boundary conditions is applied and the model 2 is obtained by applying the Fouriertransformation. It should be emphasized that this expression represents the solution at a certain time, which can be related to an axial location. Furthermore, the Levenberg-Marquardt algorithm for non-linear curve-fitting is employed to carry out the model 3 . Use of the model is restricted to the duration of injection, during which the bounds of the mixture fraction spectrums are assumed to be zero and one. Finally, the scalar dissipation rate and the mixture fracture PDF (probability density function) in the three models are calculated by using CFD (computational fluid dynamics) code and the different results are compared and evaluated. It gives the theory and method about calculating the scalar dissipation rate exactly in the non-premixed turbulent combustion.
\end{abstract}

\section{REFRENCES}

1. Reitz R.D. A temperature wall function formulation for variable-density turbulent flows with application to engine convective heat transfer modeling. Int. J. Heat Mass Transfer, 1997;40(3):613-625

2. Peters N. Turbulent Combustion, Cambridge, Cambridge University Press, 2004;128-154

3. Liu YF, Pei PC, Asymptotic analysis on autoignition and explosion limits of hydrogen-oxygen mixtures in homogeneous systems. International Journal of Hydrogen Energy, 2006; 31(5):639647 